\title{
Plane Blocks- Effective and Low Risk Procedures for Regional Analgesia and Anesthesia in Acute and Chronic Pain
}

\author{
Aron-Maor $A^{1,2}$ and Vulfsons $S^{2,3 *}$ \\ ${ }^{1}$ Meuhedet Health Services, North District, Israel \\ ${ }^{2}$ Institute for Pain Medicine, Rambam Health care Campus, Haifa, Israel \\ ${ }^{3}$ Rappaport School of Medicine, Technion Institute of Technology, Haifa, Israel
}

\begin{abstract}
Plane blocks have been developed and introduced over the last decade for perioperative anesthesia as well as for postoperative analgesia and for treatment of acute posttraumatic pain such as rib fractures. Less has been published about the use of such procedures in the management of chronic pain.

Following the successful treatment of a patient with chronic chest pain using a serratus anterior plane block, we performed a review of the available literature on the subject of plane blocks, their uses and their place in the therapeutic armamentarium available to physicians (and patients) in the field of pain medicine.

There are numerous case reports describing a variety of plane blocks having been used as an alternative to other regional anesthesia techniques (nerve plexus, epidural or paraspinal blocks), though there are no clinical head-to-head studies comparing the efficacy and safety of the plane blocks to other regional anesthesia/analgesia methods in use. We bring a review and summary of the current data available on plane blocks based on the published literature.
\end{abstract}

\section{Keywords}

Chronic pain, Plane block, TAP, PECs, Serratus anterior, Quadratus lumborum, Omohyoid, Erector spinae

\section{Introduction}

Muscles and internal organs are surrounded by fasciae these are sheets of connective tissue (mainly collagen) that surround, stabilize and separate the muscles and internal organs. The fasciae are classified by layer as superficial, visceral and deep, as well as by location and function. The potential space between fasciae is a plane into which local anesthetic can be injected, which will spread throughout the interfascial plane reaching, surrounding and blocking nerves in the area, thus achieving anesthesia or analgesia in the area (region) innervated by those nerves. Regional anesthesia is used either alone (for local surgical interventions, perioperative or post traumatic analgesia) or intraoperatively in conjunction with general anesthesia.

Plane blocks have been developed and introduced over the last decade for perioperative anesthesia as well as for postoperative analgesia and for treatment of acute posttraumatic pain such as rib fractures, though less has been published about the use of such procedures in the management of chronic pain. They are relatively easier to perform than selective nerve blocks and are gaining an increasingly important place in the clinical practice of both anesthesiologists and pain medicine practitioners. There are several plane blocks in use - corresponding to the various anatomical areas.
Following the successful treatment of a patient with chronic chest pain using a serratus anterior plane block (Figure 1), we performed a review of the available literature on the subject of plane blocks, their uses and their place in the therapeutic armamentarium available to physicians (and patients) in the field of pain medicine.

There are numerous case reports describing a variety of plane blocks having been used as an alternative to other regional anesthesia techniques (nerve plexus, epidural or paraspinal blocks), though there are no clinical head-to-head studies comparing the efficacy and safety of the plane blocks to other regional anesthesia/analgesia methods in use. We bring a review and summary of the current data available on

*Corresponding author: Simon Vulfsons, Institute for Pain Medicine, Rambam Health Care Campus; Rappaport School of Medicine, Technion Institute of Technology, P.O. Box 9602, Haifa 3109601, Israel, Tel: 972-4-7772234, Fax: 972-4-7773505

Accepted: November 24, 2020

Published online: November 26, 2020

Citation: Aron-Maor A, Vulfsons S (2020) Plane Blocks- Effective and Low Risk Procedures for Regional Analgesia and Anesthesia in Acute and Chronic Pain. Arch Fam Med Gen Pract 5(1):132-143 


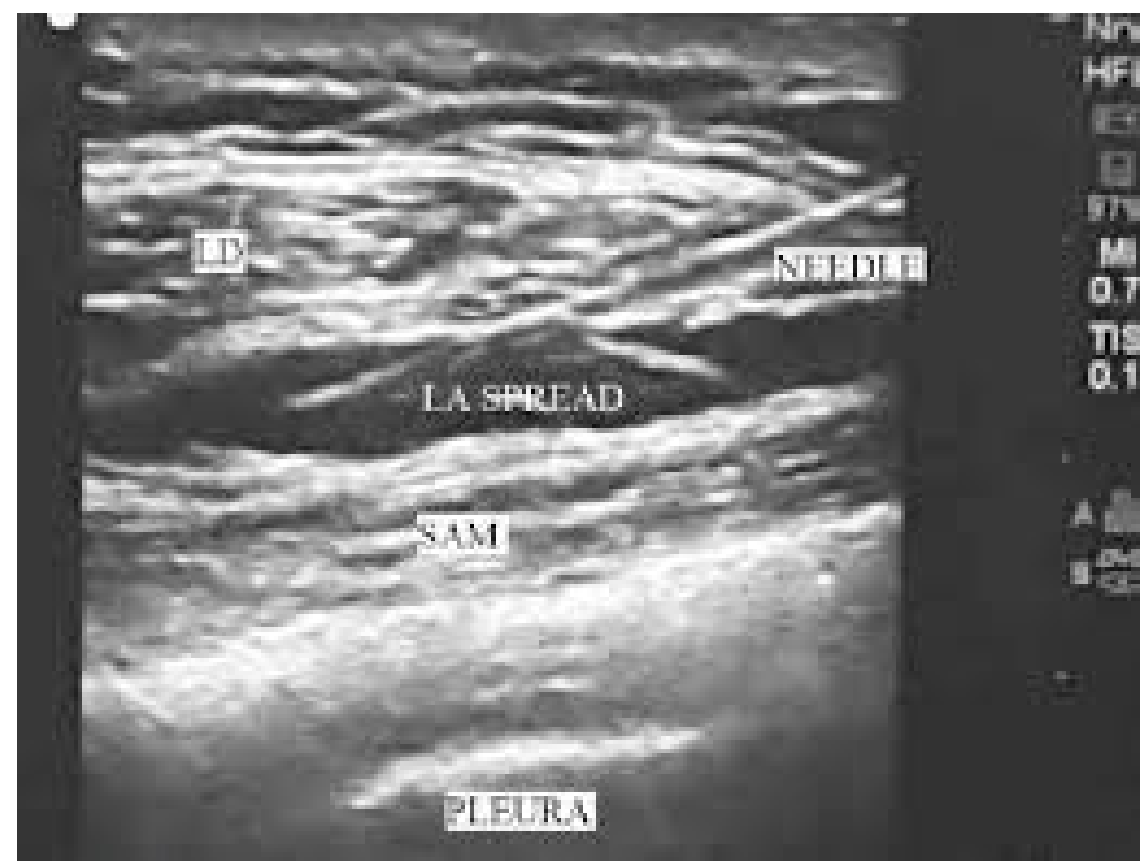

Figure 1: Sonographic image of serratus anterior block after injection of local anesthetic (LA). SAM-serratus anterior muscle, LD latissimus dorsi muscle. The tip of the needle is located below the latissimus dorsi muscle and above the serratus anterior muscle. The dark area is the local anesthetic (LA) injected between the muscles.

Source: The Student Doctor Network. forums. studentdoctor.net/threads/breast-surgery-and-serratus-anports safeterior- blocks.1263649/

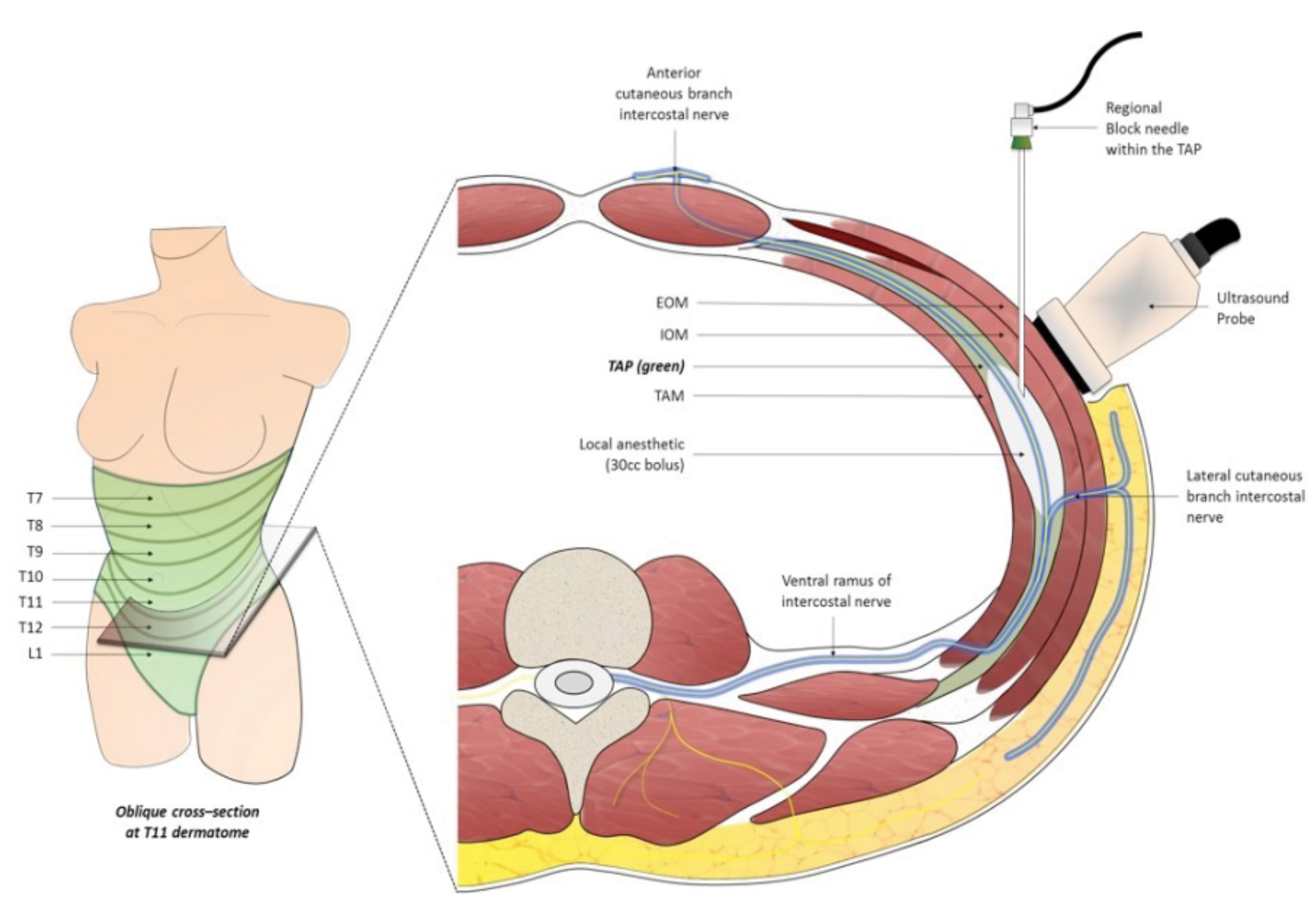

Figure 2: TAP - Transverse Abdominis Plane, EOM - external oblique muscle, IOM - internal oblique muscle, TAM - transverse abdominis muscle.

Source : Kun JM, Jablonka EM, Lamelas AM, Avraham T, Molina M, Molina B, Dayan JH, Smith ML. Transversus Abdominis Plane Blocks with Single Dose Lisosomal Bupivacaine Reduce Length os Stay Following Abdominally Based Microsurgical Breast Reconstruction. NESPS 2016 
plane blocks based on the published literature.

\section{Transversus Abdominis Plane Block}

The transversus abdominis plane (TAP) is a potential anatomical space between the transverse abdominis and the internal oblique muscles (Figure 2 and Figure 3).

The TAP block involves the injection of local anesthetic into the plane between the internal oblique and the transversus abdominis muscles. In this plane, the ventral rami of the segmental thoracolumbar nerves course before innervating the anterolateral abdominal wall.

In 2001 the method was first described [1] as a blind landmark technique using the lumbar Triangle of Petit. However the blind technique is less accurate since it relies on the subjective sensation of a "pop" sign, signaling the penetration of the fascia between the muscles. In later years, ultrasound has been used to better identify the anatomical landmarks and guide the injection of the anesthetic, thus improving the accuracy of the injection as well as its yield and efficacy.

Cadaveric studies have been performed in order to establish the spread of the injected anesthetic [2,3]. In these studies dye was injected in the TAP and subsequently dissection of the fascial planes was achieved - the spread of the dye extended from the iliac crest to the lateral border of the tenth rib in most cases. Thus the segmental nerves involved were T10 to L1 - noting that T10 was involved in only $50 \%$ percent of the cases. An additional cadaveric study using a subcostal injection technique [4] demonstrated spread of the dye to affect the T9 and T10 nerves - when a single injection was performed, and T9 to T11 nerves with multiple injections, suggesting that a subcostal TAP block may involve higher nerve roots and multiple injections may increase the spread of the injectate thus blocking more segmental nerves. Shibata and colleagues [5] assessed the extent of ultrasound guided TAP block by pinprick in 26 patients undergoing laparoscopic gynecological surgery. The mean upper and lower levels of sensory block at $30 \mathrm{~min}$ after local anesthetic injection were T10 (range, T9-11), L1 (range, T12-L1), respectively. An additional report by Hebbard [6] also describes the range of reliable analgesia with (posterior) TAP block below the umbilicus - namely in the range of T10 to L1 nerve roots. In summary we can conclude that the anatomical range of significant and reliable analgesia/anesthesia that can be achieved by a TAP block spreading from the T10 root to the L1 root levels.

The TAP block has been used both as a regional anesthesia technique intraoperatively and as a peri/post-operative analgesic modality after abdominal surgery. The surgeries during which the procedure has been used include open appendectomy [7], laparoscopic cholecystectomy [8,9], lower bowel surgery [10], after Caesarean delivery [11] and the procedure has also been described in pediatric patients [12]. The TAP block was administered in addition to general or spinal anesthesia, using either rupivacaine or levobupivacaine and in cases of major abdominal surgery (such as bowel surgery or Caesarean section) a bilateral TAP block was performed. A volume of 30-40 $\mathrm{ml}$ of injectate (on each side) was used to ensure a wide spread of the injectate and effective analgesia. In all cases the principal parameters used to assess the procedure's efficacy were opioid use in the post-operative period and VAS assessment score, and in some studies patient satisfaction with pain relief was also used as an assessment parameter.

All the studies cited reported improved postoperative analgesia as assessed by reduced opioid and other analgesics

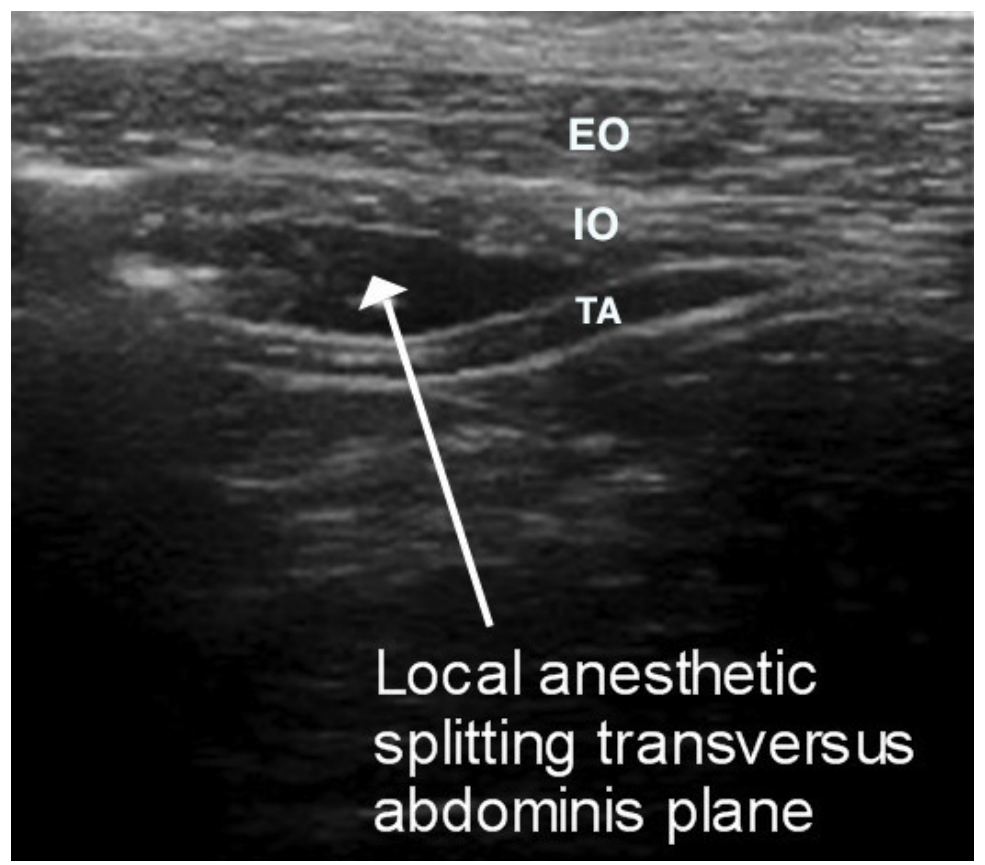

Figure 3: Ultrasound image of TAP block. EO - External Oblique, IO- Internal Oblique, TA -Transverse Abdominis. Source: Image reproduced with permission from http://pie.med.utoronto.ca/obanesthesia 
requirements in the post-operative period, lower anti-emetic treatment requirements, lower VAS scores and better satisfaction with pain relief.

However a systematic review published in 2010 [13] which included eight studies concludes there is only limited evidence to suggest that use of perioperatve TAP block reduces opioid consumption and pain scores after abdominal surgery when compared with no intervention or placebo - this despite findings of significantly less requirement for morphine 24 hours postoperative in five studies and 48 hours postoperative in one study, but not at two hours postoperative. At the time the review was published no studies existed that compared the TAP block with other analgesics such as epidural analgesia or anesthetic infiltration into the surgical abdominal wound. In 2011 a study comparing subcostal TAP catheters with epidural catheter analgesia after upper abdominal surgery in 62 patients found no significant differences the two groups regarding VAS score on coughing or at rest, failure rate or treatment success rate and it was concluded by the authors that there was no significant advantage of epidural analgesia over subcostal TAP catheter bolus analgesia in patients undergoing upper abdominal surgery - provided the incision was limited to at or above the T10 dermatome [14]. In 2014 the same group published a study of 70 patients after laparoscopic colorectal surgery for which continuous TAP analgesia was provided performing four quadrant single-shot TAP blocks followed by a continuous infusion of anesthetic via posterior TAP catheters [15] compared with thoracic epidural analgesia. In 93\% of the patients, posterior TAP catheter infusion provided dynamic postoperative analgesia comparable with thoracic epidural infusion.

An additional review of eighteen trials [16] that included diverse surgical procedures concluded that despite the rates of improved analgesia reported with TAP block postoperative "our understanding of the TAP block and its role in contempo- rary practice remains limited" and no conclusion was drawn as to the most suitable surgical procedures, dosing, timing and techniques that provide optimal analgesia following TAP block.

There are also some reports of the TAP block providing effective analgesia for visceral pain $[17,18]$ in patients with chronic recurrent pancreatitis.

Current contraindications to the TAP block include infection or malignancy at the site of the planned injection. Also, as with every invasive procedure, there are possible complications, notably intraperitoneal injection, intestinal hematoma and temporary femoral nerve block $[19,20]$. There is also a report of liver trauma secondary to ultrasound guided TAP block [21] - however these complications are rare.

In summary the TAP block appears to be an effective, safe and relatively simple procedure for peri- and postoperative analgesia, and even for surgical anesthesia in certain cases. The procedure appears to be as effective as epidural block however clinical trials that examine the safety and efficacy of the TAP block compared to established regional anesthesia techniques are still needed. We found no published literature regarding the treatment of anatomically relevant chronic pain conditions using TAP block.

\section{Pectoralis (PEC) and serratus anterior plane blocks}

Indications for performing these types of blocks include perioperative hemithoracic anesthesia/analgesia after breast surgery, after thoracic or chest wall surgery [22] or trauma (such as rib fractures) [23]. These blocks were developed as an alternative to more invasive and more potentially dangerous procedures for regional anesthesia of the chest, such as thoracic paravertebral block, intervertebral nerve block or central neuraxial blockade. The Pecs I block is used to anes-
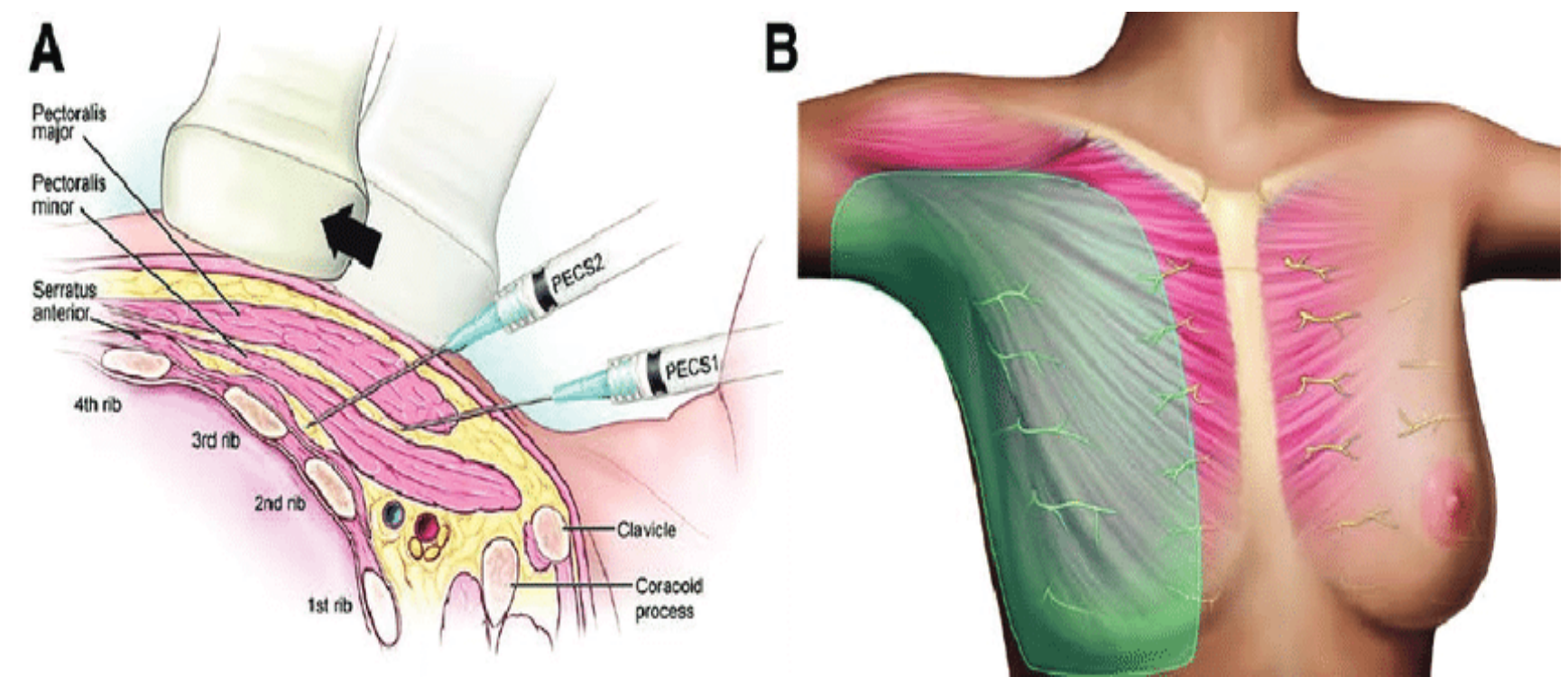

Figure 4: Injection sites for the PECS I and II plane blocks. PECS I - The needle tip is placed between the pectoralis major and pectoralis minor muscles. PECS II - The needle tip is placed between the pectoralis minor and (above) the serratus anterior muscle.

Source: Exparel.com/hcp/pec 
thetize the medial and lateral pectoral nerves that innervate the pectoralis muscles [24]. The injection is performed in the fascial plane between the pectoralis major and minor muscles (Figure 4). The Pecs II block (which includes the Pecs I block) involves a second injection (lateral to the Pecs I injection point) in the fascial plane between the pectoralis minor and serratus anterior muscles thus providing blockade of the upper intercostal nerves [25].

The serratus plane block is yet a further modification, or addition to, the Pecs I and II blocks (Figure 1 and Figure 5). The block achieves complete paresthesia of the hemithorax [24]. It can be performed at two different levels in the midaxillary line: above and below the serratus anterior muscle [25]. The injection above the muscle is performed in the fascial plane between the serratus anterior and latissimus dorsi muscles.

In a preliminary study, the superficial plane block was found to be more effective than the deep [25]. The side effect profile of these blocks so far has proven significantly better than alternative regional anesthesia methods such as intercostal nerve block [26] or thoracic paravertebral block $[27,28]$. The efficacy of these types of blocks, Pecs and serratus anterior, as assessed by time to first analgesic requirement after surgery, perioperative opioid consumption and perioperative pain was found to be an effective (and sometimes superior) alternative to thoracic spinal and paravertebral blocks $[29,30]$. In a cadaveric study conducted in order to ascertain the injectate spread in ultrasound-guided serratus anterior plane block, the lateral cutaneous branches of the intercostal nerves were found to be involved in the block, which appears to account for the analgesic effect of the block [31]. Anatomically the block was not found equivalent to the paravertebral block for rib fracture analgesia. The procedure has been used successfully to provide analgesia after a number of thoracic surgeries albeit the number of cases reported is not large and the issue requires further research [32-34].

\section{Erector-spinae plane block (ESPB)}

An additional plane block that has been described for thoracic and abdominal analgesia is the erector-spinae muscles block.

The erector-spinae muscle is actually a muscle complex composed of the triad: iliocostalis, longissimus thoracis and spinalis muscles. It originates from the sacrum and extends cranially in the paraspinal groove on either side of the spinous processes, with insertions on the thoracic and cervical vertebrae as high as C2. This muscular column is encased in a retinaculum that extends from the sacrum to the base of the cranium. In the lower back, this retinaculum is referred to as the thoracolumbar fascia. The anatomy of the ESP and its sheath provide the basis for the spread pattern of injected fluid - both cranially and caudally to surround the nerve roots above and below the level of injection. The block is performed under ultrasonographic guidance and the point of injection is usually superficial to a thoracic transverse process and deep to erector spinae muscle group (Figure 6).

In cadaveric studies investigating the mechanism of action of the ESP block there was extensive cephalocaudad and lateral spread of the dye deep and superficial to the erector spinae muscles [35]. There was no spread of the dye anteriorly to the paravertebral space - dorsal ramus involvement was posterior to the costotransverse foramen and there was potential involvement of the lateral cutaneous branches of the intercostal nerves lateral to the angle of the ribs. In a different cadaveric study designed to assess the differences between injectate spread in the ESP block versus the retrolaminar block it was observed that the ESP block produced additional spread to intercostal spaces over 5 to 9 levels which may contribute to more extensive analgesia than the retrolaminar block [36]. Some epidural and foraminal spread of the local anesthetic may also explain the clinical effect of the ESP block.

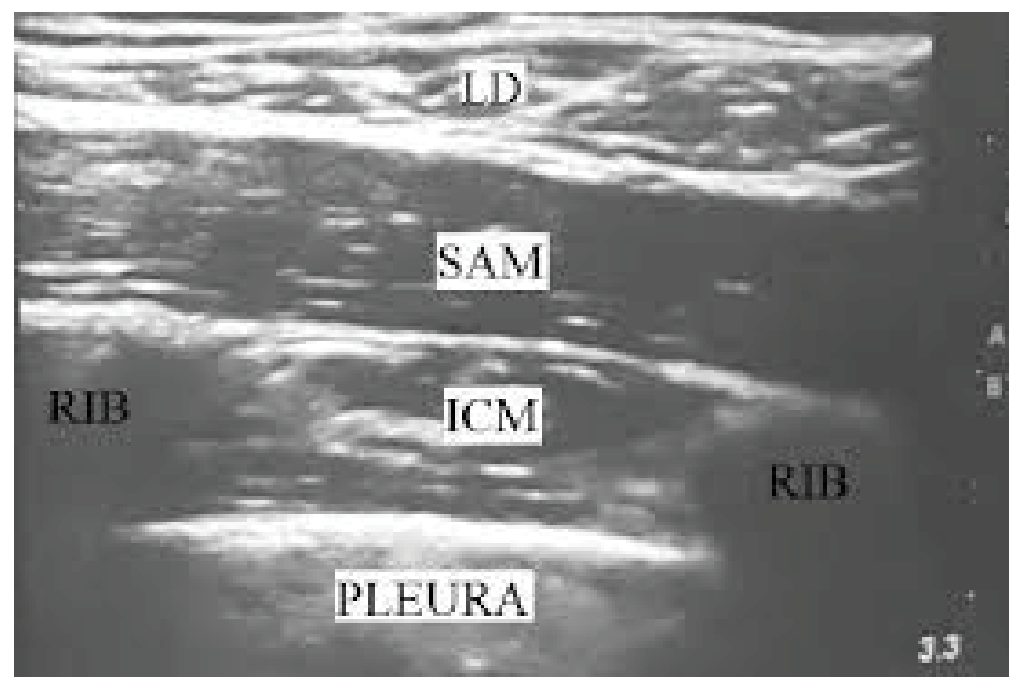

Figure 5: Sonographic image of the chest wall muscles for the serratus anterior plane block. LD: Latissimus Dorsi; SAM: Serratus Anterior Muscle; ICM: Intercostal Muscles.

Source: The Student Doctor Network. forums. studentdoctor.net/threads/breast-surgery-and-serratus-anports safeteriorblocks.1263649/ 


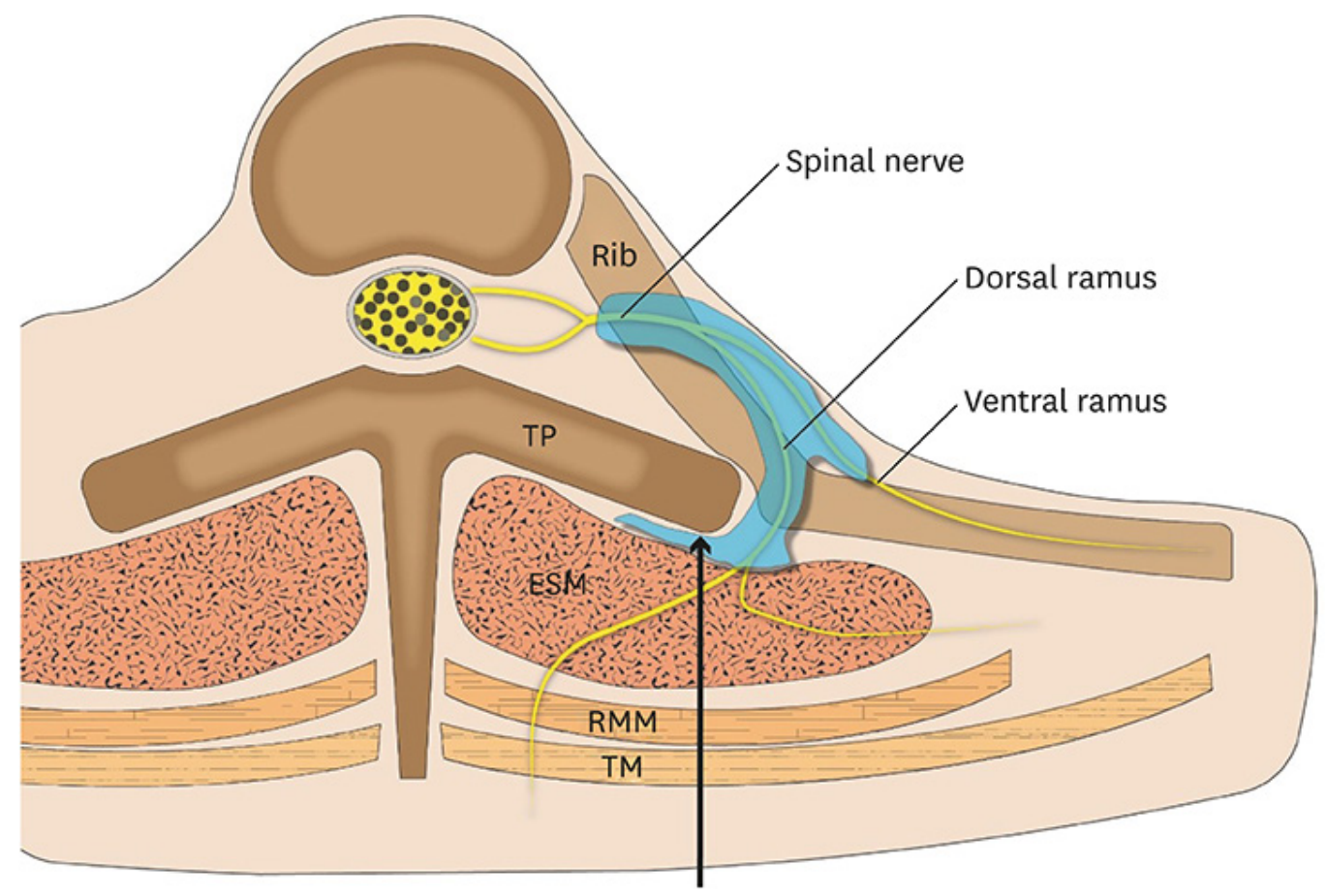

Figure 6: TP: Transverse Process; ESM: Erector Spinae Muscle; RMM: Rhomboid Major Muscle, TM: Trapezius Muscle. Blue area-spread of injectate (local anesthetic).

Source: J Korean Med Sci 2018 Nov;33(45):e291. Erector Spinae Block for effective Analgesia after Total Mastectomy with Sentinel or Axillary Lymph Node Dissection: A Report of Three Cases. Kwon WJ. Bang SU. Sun WY.

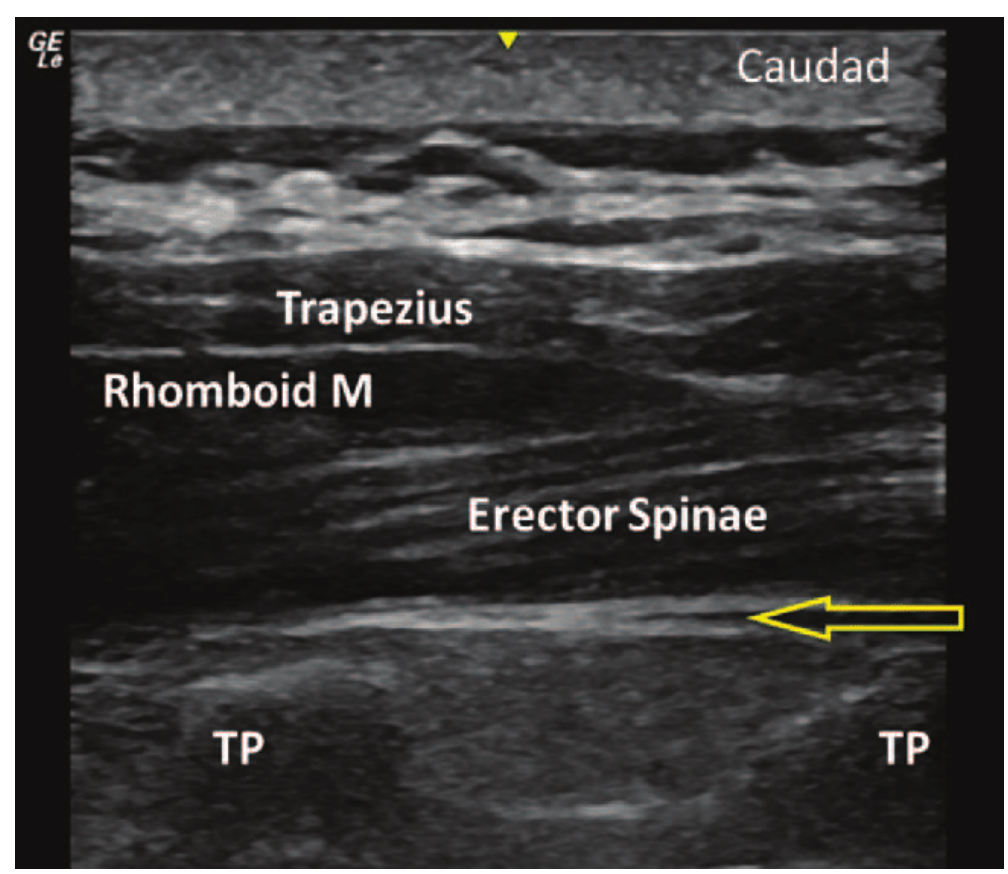

Figure 7: Sonographic image of the ESP block - arrow points to the plane between the erector spinae muscles (ESPM) and the transverse process (TP) where the local anesthetic is injected.

Source: A\&A Case Reports 2017, May. Continuous Erector Spinae Block for rescue Analgesia in Thoracotomy after Epidural Failure: A Case Report. Forrero M, Rajarathinam M, Adhikary SD, Chin JK.

In clinical reports ESP block has resulted in extensive cutaneous sensory block thus indicating both ventral and dorsal spinal rami involvement providing extensive thoracoabdomi- nal wall analgesia, with a single injection at T5 level spreading between $\mathrm{C} 7$ and T8, and even as low as L3 transverse process caudally [37] (Figure 7). 
The block has been used in the treatment of both chronic neuropathic pain [37] and in postoperative and posttraumatic pain- performed using $20-30 \mathrm{ml}$ ropivacaine (with or without steroids) [38-40].

The ESP block has been described in radical mastectomy operations, as part of multimodal intraooperative [41,42] and postoperative [43] analgesic approach. In two cases of radical mastectomy operation $[41,42]$ the method proved to be safe, easy to perform and effective both as an intraoperative analgesic modality and in having a ktpostoperative opioid sparing effect. It is notable than in the cases described by Kimachi, the procedure provided complete surgical anesthesia for the 2.5 hour surgery as the patient had high cardiovascular risk and could not be put under general anesthesia [42]. In a study of 50 randomized patients undergoing elective breast cancer surgery the block was performed preoperatively at the T4 vertebral level to all patients in the study group, whereas the patients in the control group received no intervention [43]. Morphine consumption was decreased significantly in the study group postoperatively - with a total decrease of $65 \%$ at 24 hours compared to the control group. It was concluded by the authors that the ESP block exhibits a significant analgesic effect in patients undergoing breast cancer surgery. Similar results were reported in three additional cases of minor surgery in the thoracic region in which the ESP block also proved an effective post-operative analgesic modality [44].

The ESP block has also been used successfully in thoracic surgery in pediatric patients $[45,46]$. In one case the block was performed at the end of thoracic surgery, just prior to emergence from general anesthesia, at the level of the T8 transverse process, and in another case reported the block was performed at the $\mathrm{T} 1$ transverse process pre-operative. In both cases excellent perioperative pain control was achieved with minimal anesthetic requirements and early ambulation and discharge from hospital. The ESP block proved a safe and effective regional anesthesia modality in pediatric patients. The extensive spread of the local anesthetic allowed for the injection to be performed at a distance from the incision site and still achieve wound analgesia.

Since the ESP block performed at the T5 transverse process level has been used successfully in thoracic surgical procedures, and since the spread of the anesthetic injectate has achieved an extensive anesthetized area, it was postulated that the same plane block performed at a lower thoracic vertebra level would prove effective for abdominal analgesia. In a cadaveric study, following injection at the T7 level (on both sides) there was evidence of injectate spread caudally as far as the L2-L3 transverse processes and laterally beyond the lateral border of the erector spinae muscle into the intercostal spaces at the T10-T12 levels [47]. The proximity to the origin of the lateral cutaneous branches of the thoraco-abdominal wall and the extensive caudo-cranial spread can potentially provide analgesia of the entire thoraco-abdominal wall. The bilateral ESP block performed at the lower thoracic level was used in four patients [47] undergoing laparoscopic ventral hernia repair. Although the small number of patients in this report limits drawing of conclusions as to the clinical use of the ESP block for this type of surgery, its potential as an ef- fective regional anesthetic technique in laparoscopic ventral hernia repair has been demonstrated. An additional case of lower thoracic ESP block (T8 level) for minor abdominal surgery - ileostomy closure in a high risk patient - is described with good surgical anesthesia and post-operative analgesia being achieved [48].

Local anesthetic injected into the fascial plane deep to the erector spinae muscle also penetrates anteriorly through the inter-transverse connective tissue and enters the thoracic paravertebral space where it can potentially block not only the ventral and dorsal rami of the spinal nerves, but also the rami communicants that transmit sympathetic fibers - therefore resulting in both somatic and visceral abdominal analgesia - if the injection were performed at a lower thoracic level. Laparoscopic cholecystectomy is a widely performed minimally invasive procedure with significantly reduced morbidity and mortality. The post-operative pain after this procedure, however, can be severe, involving both somatic and visceral nerve fibers. The ESP block has been used in laparoscopic cholecystectomy and better NRS scores as well as lower opioid and other rescue analgesia use were reported in the post-operative period thus demonstrating the procedure's efficacy in achieving significant visceral as well as somatic analgesia $[49,50]$. Chin and colleagues describe an additional series of three patients, after bariatric surgery, in whom bilateral lower (T7) ESP block was performed achieving significant relief of postoperative visceral pain [51]. In this case also, due to the small number of patients, drawing far reaching conclusions as to the role of ESP block as an analgesic modality in abdominal surgery is limited, however it definitely warrants further study.

The ESP block for postoperative abdominal surgery analgesia has been used and found effective in pediatric patients as well [52].

Continuous ESP block using bilateral catheters inserted into the fascial plane at the T8 level was used to achieve effective post-operative analgesia after major open lower abdominal surgery [53] thus providing a safer and simpler to perform alternative to epidural analgesia, and it has also been used in thoracotomy surgery in a pediatric patient [54].

An additional use and possible indication for the ESP block may be the management of shoulder pain. The data is sparse and we havfound so far, only two case reports - one describing the use of the procedure in the management of chronic shoulder pain [55] and the other describing the use of the ESP block in an emergency department setting for the management of neuropathic herpes zoster pain in the upper chest and shoulder region [56]. The rationale for using this technique to achieve analgesia in this high anatomical region is the fact that the erector spinae muscle extends up to the cervical spine therefore potentially being useful in painful conditions of the shoulder girdle. The cervical portion of the muscles that constitute the erector spinae muscle inserts onto the transverse processes of the $\mathrm{C} 2-\mathrm{C} 6$ vertebrae, providing a pathway for the spread of the local anesthetic. On CT imaging the injectate spread was demonstrated up to the C3 level. The procedure did prove effective in the management of 
chronic shoulder pain in the case reported [55]. Thus an ESP block performed at the T2-T3 level may exert its effect at the C5-C6 nerve roots, blocking not only the suprascapular nerve, but also the axillary and lateral pectoral nerves - potentially proving more effective than an isolated suprascapular nerve block. No comparative studies of the two procedures have been done as yet, albeit the authors [55] do report similar and successful management of several other patients with chronic shoulder pain.

The ESP block was first described in 2016. In 2019 a pooled review of 242 cases was published seeking (in the absence of controlled clinical trials) to examine the clinical data and to gain an understanding of the ESP block characteristics [57]. The authors conclusion was that the ESPB appears to be a safe and effective option for thoracic, abdominal and extremities surgeries. However despite the abundance of case reports, further clinical trials are needed to compare the effectiveness of ESPB to other regional anesthesia techniques.

Having been performed at various spinal levels in order to achieve different anatomical levels of analgesia - the ESPB has also been performed at the lumbar level (L4-L5) and has been reported to be effective in providing analgesia for hip joint and proximal femur surgeries [58,59]. However, as has been pointed out by Kose and colleagues, unlike the thoracic area where the paravertebral space boundaries are very clear, which allows for small volumes of injectate to spread cranio-caudally and affect both the ventral and the dorsal rami, achieving multi-level analgesia, in the lumbar area the paravertebral space does not have such clear boundaries [60]. Therefore the local anesthetic injected does not spread in a narrow area, but spreads anteriorly to the paravertebral space, with most of the injectate surrounding the psoas muscle and the lumbar plexus, leading to blockage of the L2-L5 nerves. Lumbar ESPB from other lumbar levels may lead to different local anesthetic spread and varying anesthetic effects. Lumbar ESPB is more similar to quadratus lumborum block.

\section{Quadratus lumborum block}

Ultrasound guided quadratus lumborum block is a relatively new regional anesthesia technique whose aim is to provide somatic analgesia for abdominal surgery.

The anatomic basis for this block lies in the layout of the myofascial compartments of the posterior abdominal wall (Figure 8).

The quadratus lumborum muscle is enclosed within the anterior and middle layers of the thoracolumbar fascia, which is a contiguous fascial system beginning at the occiput and ending at the sacrum [61]. The anterior layer of the thoracolumbar fascia is contiguous with the investing fascia of the psoas muscle medially and laterally it blends with the transversalis fascia that lines the inner aspect of the entire abdominal wall [62]. The transversalis fascia is also contiguous cranially with the endothoracic fascia, thus creating a plane between the quadratus lumborum and the transversalis fascia that can potentially permit spread of fluid from an injection

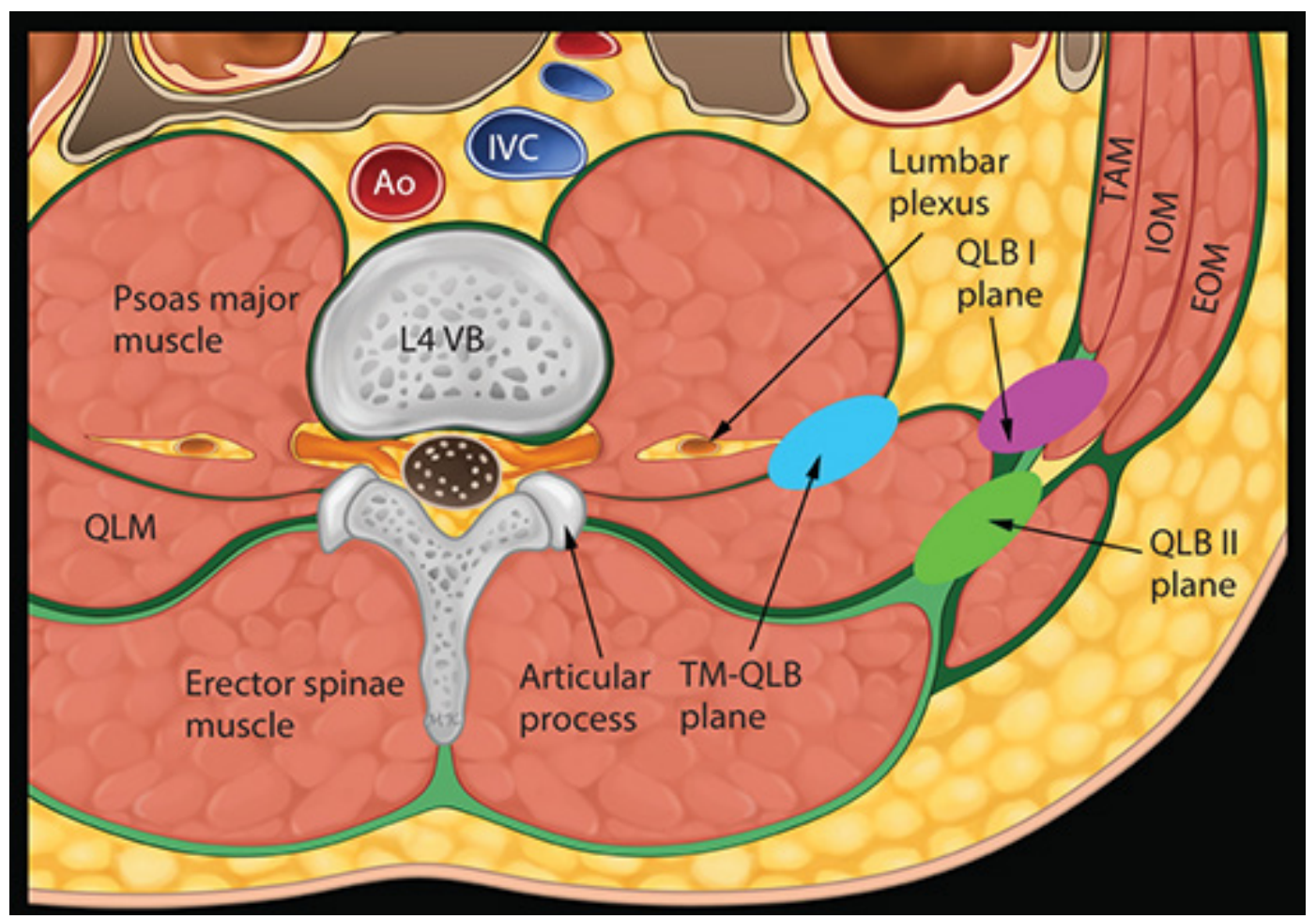

Figure 8: TAM- transverse abdominis muscle, IOM - internal oblique muscle, EOM - external oblique muscle, QLM - quadratus lumborum muscle, QLB-quadratus lumborum plane, L4VB - L4 vertebral body.

Source: Karmakar MK, Soh E, Chee V, Sheah K. Atlas of Sonoanatomy for Regional Anesthesia and Pain Medicine.

Copyright McGraw Hill Education. 
A

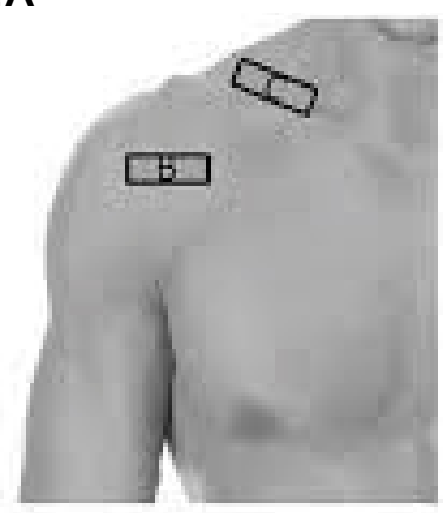

B

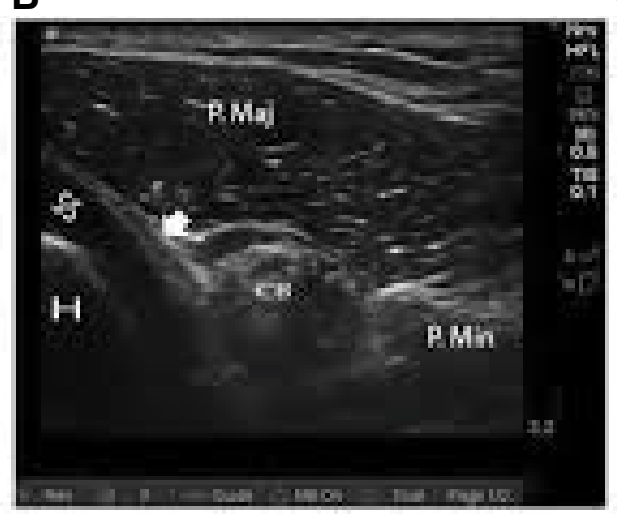

C

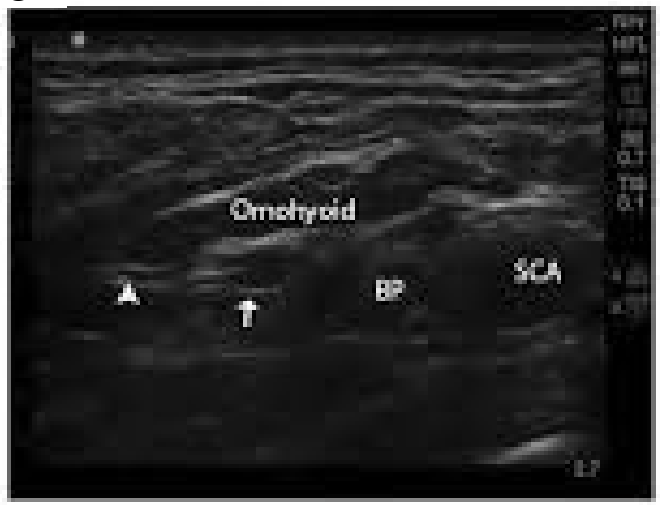

Figure 9: (A) Probe position for subscapularis plane block (B) and sub-omohyoid block (C). (b) Ultrasound image of subscapularis plane showing the lesser tuberosity of the humerus ( $\mathrm{H}$; laterally), with the attachment of the subscapularis tendon (SS) above it. The subscapularis plane is marked by the white arrowhead. At this level, the coracobrachialis (CB) and the pectoralis minor (P.Min) are seen originating from the coracoid process (not seen in this image). The pectoralis major (P.Maj) muscle is seen superficially. (C) Ultrasound image of the sub-omohyoid plane showing the inferior belly of the omohyoid muscle (omohyoid) and the adjoining suprascapular nerve (arrow) seen just lateral to the brachial plexus (BP). The target for the injection is marked by the arrowhead and is a fascial plane underneath the lateral part of the inferior belly of the omohyoid muscle. The subclavian artery (SCA) is also seen in the image.

Source: Sondekoppam RV, Lopera-Velasquez LM, Naik L, Ganapathy S. Subscapularis and sub-omohyoid plane blocks: an alternative to peripheral nerve blocks for shoulder analgesia. BJA: British Journal of Anaesthesia. 2016;117(6):831-832.

point adjacent to the quadratus lumborum muscle to both the lumbar and thoracic paravertebral spaces.

Several approaches have been described for the quadratus lumborum block [63]. The first was derived from the landmark-guided TAP block - a lateral approach to the quadratus lumborum muscle ("QL1") and was initially termed a posterior ultrasound-guided TAP block [3]. An anterior approach has also been described where the needle is inserted into the plane between the quadratus lumborum muscle and the transversalis fascia through the muscle in a posterior-anterior approach [64] and finally a posterior QL block ("QL2") in which the injection is performed on the posterior surface of the quadratus lumborum muscle instead of on the ventral aspect [65]. The block has been used for postoperative analgesia after a number of abdominal surgeries with better pain control reported and reduced opioid consumption [66-70]. Somatic analgesia extended mostly between T7-L1 dermatomes also involving the hip and groin region. Based on these clinical reports as well as cadaveric studies the quadratus lumborum block may prove an acceptable alternative to lumbar plexus block for perioperative analgesia in abdominal and hip and inguinal region surgeries [71]. Bilateral continuous quadratus lumborum block has been described as an effective means to achieve prolonged postoperative analgesia after major abdominal surgery [72].

\section{Sub-omohyoid plane block}

The sub-omohyoid plane block is a regional anesthesia technique used for shoulder anesthesia. The shoulder joint and its muscles are entirely innervated by the brachial plexus - the block that is considered the "gold standard" for regional shoulder anesthesia is the interscalene block which, while effective, has potentially serious complications including neurologic damage, intrathecal spread and systemic toxicity of local anesthetic [73-75]. Blocking of distal nerves, while safer, is more cumbersome since in order to achieve effective analgesia (especially for major surgery of the shoulder) it is necessary to block in addition to the axillary and suprascapular nerves, the infraclavicular and subscapular nerves. This requires multiple injections which cause more patient discomfort. The suprascapular nerve leaves the superior trunk of the brachial plexus close to the supraclavicular fossa and travels in the posterior triangle of the neck parallel to the inferior belly of the omohyoid muscle to enter the suprascapular notch [76]. The axillary nerve leaves the posterior cord of the brachial plexus in the infraclavicular fossa and splits into the anterior and the posterior branches, crossing the subscapularis muscle before winding around the humerus to enter the quadrangular space. The subscapular nerves arising from the posterior cord are also found on the ventral surface of the subscapularis muscle [77]. We found two variations of the sub omohyoid plane block described. The first was reported by Sondekoppam and colleagues who perform combined sub-omohyoid plane block together with a subscapularis plane block to achieve shoulder analgesia with minimal impact on phrenic nerve function [78] (Figure 9). The local anesthetic is placed over the subscapularis muscle thereby blocking both the axillary and the subscapular nerves, after which and additional amount of local anesthetic is deposited over the clavicle under the inferior belly of the omohyoid muscle to block the suprascapular nerve. Another combination block for shoulder analgesia/anesthesia was described involving a single puncture technique covering both the infraclavicular and suprascapular nerves, covering the entire innervation of the shoulder joint and muscles without compromising diaphragmatic function [79].

\section{Summary}

Pain is one of the most, if not the most, common com- 
plaints in the human condition. The management of pain be it acute or chronic, is an ongoing challenge for the medical profession, with a limited number of treatment modalities available and often further limited by side effects and complications thereof. From amongst the invasive techniques in use for the management of pain, regional anaesthesia/analgesia procedures have proven useful for both postoperative (acute) and chronic pain. The advent of ultrasound to guide these procedures has increased our ability to achieve more accuracy and more effective pain control. The plane blocks described in this review are anatomy-based developed techniques that are easier to perform than, for example, plexus nerve blocks or peripheral nerve blocks and have fewer and milder possible complications and side effects. They represent important tools in the pain management clinician`s armamentarium especially since many of those blocks can be performed in an outpatient ambulatory setting. More studies are required to assess their efficacy compared to other regional blocks in use today for perioperative analgesia as well as their role in the management of chronic pain conditions.

\section{References}

1. Rafi A (2001) Abdominal field block: A new approach via the lumbar triangle. Anaesthesia 56: 1024-1026.

2. Tran TMN, Ivanusic JJ, Hebbard P, et al. (2008) Determination of spread of injectate after ultrasound-guided transversus abdominis plane block: A cadaveric study. British Journal of Anaesthesia 102: 123-127.

3. Carney J, Finnerty O, Rauf J, et al. (2011) Studies on the spread of local anaesthetic solution in transversus abdominis plane blocks. Anaesthesia 66: 1023-1030.

4. Barrington MJ, Ivanusic JJ, Rozen WM, et al. (2009) Spread of injectate after ultrasound-guided subcostal transversus abdominis plane block: A cadaveric study. Anaesthesia 64: 745-750.

5. Shibata $Y$, Sato $Y$, Fujiwara $Y$, et al. (2007) Transversus abdominis plane block. Anesthesia \& Analgesia 105: 883.

6. Hebbard P (2008) Subcostal transversus abdomin is plane block under ultrasound guidance. Anesthesia \& Analgesia 106: 674675.

7. Niraj G, Searle A, Mathews M, et al. (2009) Analgesic efficacy of ultrasound-guided transversus abdominis plane block in patients undergoing open appendicectomy ${ }^{\dagger}$. British Journal of Anaesthesia 103: 601-605.

8. El-Dawlatly, Turkistani A, Kettner S, et al. (2009) Ultrasound-guided transversus abdominis plane block: Description of a new technique and comparison with conventional systemic analgesia during laparoscopic cholecystectomy. Br J Anaesth 102: 763-767.

9. Ra YS, Kim CH, Lee GY, et al. (2010) The analgesic effect of the ultrasound-guided transverse abdominis plane block after laparoscopic cholecystectomy. Korean J Anesthesiol 58: 362-368.

10. Hebbard P, Fujiwara Y, Shibata Y, et al. (2007) Ultrasound-guided transversus abdominis plane (TAP) block. Anaesth Intensive Care 35: 616-618.

11. Belavy D, Cowlishaw PJ, Howes M, et al. (2009) Ultrasound-guided transversus abdominis plane block for analgesia after Caesarean delivery. British Journal of Anaesthesia 103: 726-730.

12. Suresh S, Chan VW (2009) Ultrasound guided transversus abdominis plane block in infants, children and adolescents: A sim- ple procedural guidance for their performance. Pediatric Anesthesia 19: 296-299.

13. Charlton S, Cyna AM, Middleton P, et al. (2010) Perioperative transversus abdominis plane (TAP) blocks for analgesia after abdominal surgery. Cochrane Database Syst Rev.

14. Niraj G, Kelkar A, Jeyapalan I, et al. (2011) Comparison of analgesic efficacy of subcostal transversus abdominis plane blocks with epidural analgesia following upper abdominal surgery. Anaesthesia 66: 465-471.

15. Niraj G, Kelkar A, Hart E, et al. (2014) Comparison of analgesic efficacy of four-quadrant transversus abdominis plane (TAP) block and continuous posterior TAP analgesia with epidural analgesia in patients undergoing laparoscopic colorectal surgery: An openlabel, randomised, non-inferiority trial. Anaesthesia 69: 348-355.

16. Abdallah FW, Chan VW, Brull R (2012) Transversus abdominis plane block: A systematic review. Regional Anesthesia and Pain Medicine 37: 193-209.

17. Smith DI, Hoang K, Gelbard W (2014) Treatment of acute flares of chronic pancreatitis pain with ultrasound guided transversus abdominis plane block: A novel application of a pain management technique in the acute care setting. Case reports in emergency medicine.

18. Smith DI, Hawson A, Correll L (2015) Transversus abdominis plane block and treatment of viscerosomatic abdominal pain. Regional anesthesia and pain medicine 40: 731-732.

19. Baeriswyl M, Kirkham KR, Kern C, et al. (2015) The analgesic efficacy of ultrasound-guided transversus abdominis plane block in adult patients: A meta-analysis. Anesthesia \& Analgesia 121: 1640-1654.

20. Salaria ON, Kannan M, Kerner B, et al. (2017) A rare complication of a tap block performed after caesarean delivery. Case reports in anesthesiology.

21. Lancaster P, Chadwick M (2010) Liver trauma secondary to ultrasound-guided transversus abdominis plane block. British Journal of Anaesthesia 104: 509-510.

22. Purcell N, Wu D (2014) Novel use of the PECS II block for upper limb fistula surgery. Anaesthesia 69: 1294-1294.

23. Blanco R (2011) The 'pecs block': A novel technique for providing analgesia after breast surgery. Anaesthesia 66: 847-848.

24. Blanco R, Fajardo M, Maldonado TP (2012) Ultrasound description of Pecs II (modified Pecs I): A novel approach to breast surgery. Rev Esp Anestesiol Reanim 59: 470-475.

25. Behnke H, Worthmann F, Cornelissen J, et al. (2002) Plasma concentration of ropivacaine after intercostal blocks for video-assisted thoracic surgery. British Journal of Anaesthesia 89: 251253.

26. Blanco R, Parras T, McDonnell JG, et al. (2013) Serratus plane block: a novel ultrasound-guided thoracic wall nerve block. Anaesthesia 68: 1107-1113.

27. Schnabel A, Reichl S, Kranke P, et al. (2010) Efficacy and safety of paravertebral blocks in breast surgery: A meta-analysis of randomized controlled trials. British Journal of Anaesthesia 105: 842-852.

28. Norum HM, Breivik H (2011) Thoracic paravertebral blockade and thoracic epidural analgesia: Two extremes of a continuum. Anesthesia \& Analgesia 112: 990.

29. Versyck B, Houwe PV, van Geffen GJ, et al. (2017) A qualitative 
Citation: Aron-Maor A, Vulfsons S (2020) Plane Blocks- Effective and Low Risk Procedures for Regional Analgesia and Anesthesia in Acute and Chronic Pain. Arch Fam Med Gen Pract 5(1):132-143

systematic review of the pectoral nerves block type I and II. Acta Anaesthes Bel 68: 49-62.

30. Khalil AE, Abdallah NM, Bashandy GM, et al. (2017) Ultrasound-guided serratus anterior plane block versus thoracic epidural analgesia for thoracotomy pain. Journal of Cardiothoracic and Vascular Anesthesia 31: 152-158.

31. J Mayes, E Davison, P Panahi, et al. (2016) An anatomical evaluation of the serratus anterior plane block. Anaesthesia.

32. Zocca JA, Chen GH, Puttanniah VG, et al. (2017) Ultrasound-guided serratus plane block for treatment of postmastectomy pain syndromes in breast cancer patients: A case series. Pain Practice 17: 141-146.

33. Khemka R, Chakraborty A, Ahmed R, et al. (2016) Ultrasound-guided serratus anterior plane block in breast reconstruction surgery. A A Case Rep 6: 280-282.

34. Madabushi R, Tewari S, Gautam SK, et al. (2015) Serratus anterior plane block: A new analgesic technique for post-thoracotomy pain. Pain Physician 18: E421-424.

35. Ivanusic J, Konishi Y, Barrington MJ (2018) A cadaveric study investigating the mechanism of action of erector spinae blockade. Reg Anesth Pain Med 43: 567-571.

36. Adhikary SD, Bernard S, Lopez H, et al. (2018) Erector spinae plane block versus retrolaminar block: A magnetic resonance imaging and anatomical study. Reg Anesth Pain Med 43: 756-762.

37. Forero $M$, Adhikary SD, Lopez $\mathrm{H}$, et al. (2016) The erector spinae plane block: A novel analgesic technique in thoracic neuropathic pain. Regional Anesthesia and Pain Medicine 41: 621-627.

38. Adhikary SD, Pruett A, Forero M, et al. (2018) Erector spinae plane block as an alternative to epidural analgesia for post-operative analgesia following video-assisted thoracoscopic surgery: A case study and a literature review on the spread of local anaesthetic in the erector spinae plane. Indian J Anaesth 62: 75-78.

39. Forero M, Rajarathinam M, Adhikary S, et al. (2017) Erector spinae plane (ESP) block in the management of post thoracotomy pain syndrome: A case series. Scand J Pain 17: 325-329.

40. Forero M, Rajarathinam M, Adhikary S, et al. (2017) Continuous erector spinae plane block for rescue analgesia in thoracotomy after epidural failure: A case report. A A Case Rep 8: 254-256.

41. Veiga M, Costa D, Brazão I (2018) Erector spinae plane block for radical mastectomy: A new indication? Rev Esp Anestesiol Reanim 65: 112-115.

42. Kimachi PP, Martins EG, Peng P, et al. (2018) The erector spinae plane block provides complete surgical anesthesia in breast surgery: A case report. A A Pract 11: 186-188.

43. Gürkan Y, Aksu C, Kuş A, et al. (2018) Ultrasound guided erector spinae plane block reduces postoperative opioid consumption following breast surgery: A randomized controlled study. J Clin Anesth 50: 65-68.

44. Balaban O, Aydin T, Yaman M (2018) Is ultrasound guided erector spinae plane block sufficient for surgical anesthesia in minor surgery at thoracal region? J Clin Anesth 47: 7-8.

45. Muñoz F, Cubillos J, Bonilla AJ, et al. (2017) Erector spinae plane block for postoperative analgesia in pediatric oncological thoracic surgery. Can J Anaesth 64: 880-882.

46. Hernandez MA, Palazzi L, Lapalma J, et al. (2018) Erector spinae plane block for surgery of the posterior thoracic wall in a pediatric patient. Reg Anesth Pain Med 43: 217-219.
47. Chin KJ, Adhikary S, Sarwani N, et al. (2017) The analgesic efficacy of pre-operative bilateral erector spinae plane (ESP) blocks in patients having ventral hernia repair. Anaesthesia 72: 452-460.

48. Tulgar S, Thomas DT, Deveci U (2018) Erector spinae plane block provides sufficient surgical anesthesia for ileostomy closure in a high-risk patient. J Clin Anesth 48: 2-3.

49. Hannig KE, Jessen C, Soni UK, et al. (2018) Erector spinae plane block for elective laparoscopic cholecystectomy in the ambulatory surgical setting. Case Rep Anesthesiol.

50. Tulgar S, Kapakli MS, Senturk O, et al. (2018) Evaluation of ultrasound-guided erector spinae plane block for postoperative analgesia in laparoscopic cholecystectomy: A prospective, randomized, controlled clinical trial. J Clin Anesth 49: 101-106.

51. Chin KJ, Malhas L, Perlas A (2017) The erector spinae plane block provides visceral abdominal analgesia in bariatric surgery: A report of 3 cases. Reg Anesth Pain Med 42: 372-376.

52. Aksu C, Gürkan Y (2018) Ultrasound guided erector spinae block for postoperative analgesia in pediatric nephrectomy surgeries. J Clin Anesth 45: 35-36.

53. Restrepo-Garces CE, Chin KJ, Suarez P, et al. (2017) Bilateral continuous erector spinae plane block contributes to effective postoperative analgesia after major open abdominal surgery: A case report. A A Case Rep 9: 319-321.

54. Wong J, Navaratnam M, Boltz G, et al. (2018) Bilateral continuous erector spinae plane blocks for sternotomy in a pediatric cardiac patient. J Clin Anesth 47: 82-83.

55. Forero M, Rajarathinam M, Adhikary SD, et al. (2018) Erector spinae plane block for the management of chronic shoulder pain: A case report. Canadian Journal of Anesthesia/Journal canadien d'anesthésie 65: 288-293.

56. Tekin E, Ahiskalioglu A, Aydin ME, et al. (2019) High-thoracic ultrasound-guided erector spinae plane block for acute herpes zoster pain management in emergency department. Am J Emerg Med 37: 375.e1-375.e3.

57. Tsui BCH, Fonseca A, Munshey F, et al. (2019) The erector spinae plane (ESP) block: A pooled review of 242 cases. J Clin Anesth 53: 29-34.

58. Tulgar S, Senturk O (2018) Ultrasound guided Erector Spinae Plane block at L-4 transverse process level provides effective postoperative analgesia for total hip arthroplasty. J Clin Anesth 44: 68 .

59. Tulgar S, Selvi O, Senturk O, et al. (2018) Clinical experiences of ultrasound-guided lumbar erector spinae plane block for hip joint and proximal femur surgeries. J Clin Anesth 47: 5-6.

60. Kose HC, Kose SG, Thomas DT (2018) Lumbar versus thoracic erector spinae plane block: Similar nomenclature, different mechanism of action. J Clin Anesth 48: 1.

61. Mirilas P, Skandalakis JE (2009) Surgical anatomy of the retroperitoneal spaces-part I: Embryogenesis and anatomy. Am Surg 75: 1091-1097.

62. Willard FH, Vleeming A, Schuenke MD, et al. (2012) The thoracolumbar fascia: Anatomy, function and clinical considerations. J Anat 221: 507-536.

63. El-Boghdadly K, Elsharkawy H, Short A, et al. (2016) Quadratus lumborum block nomenclature and anatomical considerations. Reg Anesth Pain Med 41: 548-549.

64. Børglum J, Moriggl B, Jensen K, et al. (2013) Ultrasound-guided 
transmuscular quadratus lumborum blockade. Br J Anaesth.

65. Blanco R, McDonnell J (2014) Optimal point of injection: The quadratus lumborum type I and II blocks. Anaesthesia.

66. Blanco R, Ansari T, Girgis E (2015) Quadratus lumborum block for postoperative pain after caesarean section: A randomised controlled trial. European Journal of Anaesthesiology (EJA) 32: 812-818.

67. Carvalho R, Segura E, do Céu Loureiro M, et al. (2017) Quadratus lumborum block in chronic pain after abdominal hernia repair: Case report. Brazilian Journal of Anesthesiology (English Edition) 67: 107-109.

68. Sebbag I, Qasem F, Dhir S (2017) Ultrasound guided quadratus lumborum block for analgesia after cesarean delivery: Case series. Revista brasileira de anestesiologia 67: 418-421.

69. Murouchi T, Iwasaki S, Yamakage M (2016) Quadratus lumborum block: Analgesic effects and chronological ropivacaine concentrations after laparoscopic surgery. Regional Anesthesia and Acute Pain: Original Articles 41.

70. Kadam VR (2013) Ultrasound-guided quadratus lumborum block as a postoperative analgesic technique for laparotomy. Journal of anaesthesiology, clinical pharmacology 29: 550.

71. Adhikary SD, El-Boghdadly K, Nasralah Z, et al. (2017) A radiologic and anatomic assessment of injectate spread following transmuscular quadratus lumborum block in cadavers. Anaesthesia 72: 73-79.

72. Shaaban M, Esa WAS, Maheshwari K, et al. (2015) Bilateral con- tinuous quadratus lumborum block for acute postoperative abdominal pain as a rescue after opioid-induced respiratory depression. A \& A case reports 5: 107-111.

73. Stundner O, Meissnitzer M, Brummett CM, et al. (2016) Comparison of tissue distribution, phrenic nerve involvement, and epidural spread in standard-vs low-volume ultrasound-guided interscalene plexus block using contrast magnetic resonance imaging: A randomized, controlled trial. Br J Anaesth 116: 405-412.

74. Mian A, Chaudhry I, Huang R, et al. (2014) Brachial plexus anesthesia: A review of the relevant anatomy, complications, and anatomical variations. Clinical Anatomy 27: 210-221.

75. Conroy PH, Awad IT (2011) Ultrasound-guided blocks for shoulder surgery. Curr Opin Anaesthesiol 24: 638-643.

76. Panero AJ, Hirahara AM (2016) A guide to ultrasound of the shoulder, part 2: The diagnostic evaluation. Am J Orthop (Belle Mead NJ) 45: 233-238.

77. Aszmann OC, Dellon AL, Birely BT, et al. (1996) Innervation of the human shoulder joint and its implications for surgery. Clinical Orthopaedics and Related Research 330: 202-207.

78. Sondekoppam RV, Lopera-Velasquez LM, Naik L, et al. (2016) Subscapularis and sub-omohyoid plane blocks: An alternative to peripheral nerve blocks for shoulder analgesia. BJA: British Journal of Anaesthesia 117: 831-832.

79. Taha AM (2017) ISO (Infraclavicular-SubOmohyoid) block: A single-puncture technique for diaphragm-and opioid-sparing shoulder anaesthesia. BJA: British Journal of Anaesthesia 119: 170-171.

DOI: $10.36959 / 577 / 495$

Copyright: (C) 2020 Aron-Maor A, et al. This is an open-access article distributed under the terms of the Creative Commons Attribution License, which permits unrestricted use, distribution, and reproduction in any medium, provided the original author and source are credited. 